\title{
Prevalence and predictors of post-traumatic stress symptoms in adolescent and young adult cancer survivors: a I-year follow-up study
}

\author{
Minyoung Kwak'*, Brad J. Zebrack', Kathleen A. Meeske², Leanne Embry ${ }^{3}$, Christine Aguilar ${ }^{3}$, Rebecca Block ${ }^{4}$, \\ Brandon Hayes-Lattin ${ }^{4}$, Yun Li, ${ }^{5,6}$, Melissa Butler ${ }^{7}$ and Steven Cole ${ }^{8}$ \\ 'School of Social Work, University of Michigan, Ann Arbor, MI, USA \\ ${ }^{2}$ Children's Hospital Los Angeles, University of Southern California, Los Angeles, CA, USA \\ ${ }^{3}$ University of Texas Health Science Center, San Antonio, TX, USA \\ ${ }^{4}$ Oregon Health and Sciences University, Portland, OR, USA \\ ${ }^{5}$ Department of Biostatistics, University of Michigan, Ann Arbor, MI, USA \\ ${ }^{6}$ Cancer Surveillance and Outcomes Research Team, University of Michigan Comprehensive Cancer Center, Ann Arbor, MI, USA \\ ${ }^{7}$ Department of Behavioral Science, University of Michigan, Dearborn, MI, USA \\ ${ }^{8}$ Hopelab Foundation, Redwood City, CA, USA
}

*Correspondence to:

School of Social Work, University of Michigan, 1080 S. University, Ann Arbor, MI, 48109-1 106, USA.E-mail: mykwak@umich. edu

Received: 30 March 2012 Revised: 24 September 2012 Accepted: 10 October 2012

\begin{abstract}
Objectives: Post-traumatic stress symptoms (PTSS) have been identified as a meaningful indicator of distress in cancer survivors. Distinct from young adult survivors of childhood cancer, young people diagnosed with cancer as adolescents and young adults (AYAs) face unique psychosocial issues; however, there is little published research of PTSS in the AYA population. This study examines prevalence and predictors of PTSS among AYAs with cancer.

Methods: As part of a longitudinal study of AYAs with cancer, 151 patients aged 15-39 years completed mailed surveys at 6 and 12 months post-diagnosis. Severity of PTSS was estimated at 6 and 12 months post-diagnosis. Multiple regression analyses were conducted to investigate the predictive effects of socio-demographic and clinical characteristics on changes in PTSS over time.

Results: At 6 and 12 months, respectively, 39\% and 44\% of participants reported moderate to severe levels of PTSS; 29\% had PTSS levels suggestive of post-traumatic stress disorder. No significant differences in severity of PTSS between 6 and 12 months were observed. Regression analyses suggested that a greater number of side effects were associated with higher levels of PTSS at 6 months. Currently receiving treatment, having surgical treatment, diagnosis of a cancer type with a $\mathbf{9 0 - 1 0 0 \% ~ s u r v i v a l ~ r a t e , ~}$ remaining unemployed/not in school, and greater PTSS at 6 months were associated with higher levels of PTSS at 12 months.

Conclusions: Post-traumatic stress symptoms were observed as early as 6 months following diagnosis and remained stable at 12-month follow-up. The development of early interventions for reducing distress among AYA patients in treatment is recommended.

Copyright @ 2012 John Wiley \& Sons, Ltd.
\end{abstract}

\section{Introduction}

Diagnosis and treatment of cancer constitute stressful and life-threatening events. A growing body of literature has examined traumatic qualities of cancer that relate to posttraumatic stress symptoms (PTSS) and post-traumatic stress disorder (PTSD) [1-3]. The experience of cancer includes multiple and chronic stressors related to threatening life and bodily integrity, such as diagnosis, severity of cancer, invasive procedures, treatment side effects, risk of relapse, and interference with physical and social functioning $[4,5]$. For cancer patients, these stressors may emerge repeatedly for an extended period. Because of these unique characteristics of cancer as both acute (e.g. diagnosis) and chronic (e.g. treatment) stressors, symptoms of posttraumatic stress may emerge differentially over the course of cancer.

Post-traumatic stress symptoms include re-experiencing of the traumatic event, such as intrusive memories and nightmares; avoidance of thoughts, feelings, and reminders related to the trauma and emotional numbing; and persistent arousal, such as hyper-vigilance and insomnia [6]. In addition to exposure to a traumatic event, a diagnosis of PTSD requires the presence of the above-described PTSS symptoms, along with two additional criteria: PTSS persist for at least 1 month after trauma; and PTSS symptoms manifest physical symptoms and significant impairment to individuals' functioning [6]. When patients suffer from these symptoms, they may have difficulties in attending to appointments or understanding information related to medical care during visits [7]. This study focuses on the prevalence and predictors of PTSS over 1 year among adolescents and young adults (AYAs) recently diagnosed with cancer, an understudied population who may be receiving inadequate psychosocial care.

Adolescents and young adults who are diagnosed with cancer during their teenage or young adult years are at risk for distress because they face unique challenges resulting from coping with both their current developmental stage and a life-threatening illness [8-11]. For example, AYAs 
with cancer experience disruptions in psychological and social maturation, diminished autonomy in relationships with their parents, stifled educational attainment and employment, and concerns related to sexuality and fertility, social isolation, and physical changes resulting from treatment [8-10,12-14]. Indeed, patients diagnosed with cancer in adolescence and young adulthood experience greater distress when compared with younger patients or older adult patients with cancer [15-18]. Research on PTSD and PTSS in the general population suggests that adolescents are particularly vulnerable to PTSD because the experience of trauma disrupts the ability to achieve key development tasks such as developing a sense of identity [19].

Despite the potential for great distress and PTSS in AYAs, few studies have examined the prevalence and predictors of PTSS in AYAs. Most studies of cancerrelated PTSS for this age group have focused on adolescent and young adult survivors of childhood cancer. A series of studies of adolescent and young adult survivors of childhood cancer indicate that $3-21 \%$ of respondents report clinically significant levels of PTSS [1,20-33]. Considering that these studies are based on long-term survivors of childhood cancer who are between 1 and 34 years post-diagnosis, the severity of PTSS may be higher if closer to the time of diagnosis or treatment. Studies suggest that PTSS are likely to be higher for those who are receiving active treatment when compared with those who are not [34,35]. For example, Phipps et al. [35] observed differences in reporting PTSS over time, with higher levels of PTSS observed in respondents who were within 2 years of diagnosis compared with those who were 2 to 5 years after completion of treatment. These studies suggest that patients in treatment or early phases of survivorship may experience more severe PTSS than longterm survivors. Thus, it is crucial to understand to what extent AYA patients with cancer experience PTSS in early phases of survivorship in order to intervene early to reduce the risk of PTSS and subsequent maladaptive outcomes over time.

Risk factors for PTSS in childhood cancer survivors include older age at diagnosis, female gender, unemployment, lower levels of educational attainment, and severe late effects or health problems $[1,27,29,31,36]$. In addition, studies of AYAs with cancer have observed greater psychological distress in those who were female, older, non-White, off treatment, and those who reported pain, treatment-related side effects, and lower levels of physical functioning [37-40].

The present study aims (i) to identify the prevalence of PTSS at 6 and 12 months post-diagnosis and the risk of PTSD at 12 months; (ii) to assess whether the prevalence of PTSS changes over the first year of diagnosis; and (iii) to examine which demographic and health status variables relate to changes in PTSS.

\section{Method}

Design, procedure, and participants

As part of a longitudinal study to observe changes in psychological adaptation, quality of life, and health service utilization over 2 years in AYA patients who were recently diagnosed with cancer, the present study used data collected within the first year of the survey. Baseline data were collected within the first 4 months of diagnosis and, subsequently, at 6 and 12 months after the baseline survey. Participants were recruited from five tertiary care academic medical centers (three children's hospitals and two university-affiliated adult care medical institutions) in Los Angeles (California), Portland (Oregon), and San Antonio (Texas) between March 2008 and April 2010. Inclusion criteria were (i) diagnosis between the ages of 14 and 39 years (but anticipated to turn 15 years while in treatment), (ii) first diagnosis of and treatment for cancer, and (iii) ability to read and understand English or Spanish.

Institutional review board approval from each participating site and the coordinating center was obtained. Research staff in clinics identified 286 eligible patients and sought physician approval for recruitment. Fifty-eight patients were dropped because either physicians denied access to patients who in their estimation were too sick to participate or patients refused to participate. Of 228 AYAs recruited and consented to study, 12 did not return a completed survey after providing consent, and one died. Thus, the overall participation rate was $75 \%(n=215)$. Subjects received $\$ 25$ incentive for completing the questionnaire at each time point. Patient assent and parent permission were obtained for those under age 18 years.

\section{Measure}

\section{Dependent outcome}

Post-traumatic stress symptoms: Post-traumatic stress symptoms were assessed using the Posttraumatic Stress Diagnostic Scale (PDS) [41] at 6-month follow-up (Cronbach's $\alpha$ 0.91) and again at 12-month follow-up (Cronbach's $\alpha$ 0.92). This measure includes 17 questions covering three categories of symptoms: re-experiencing (five items), avoidance (seven items), and arousal (five items). We adapted the items in the PDS by replacing the word 'trauma' with 'cancer' in order to specifically investigate cancer-related PTSS. Each symptom was rated on a scale of 0-3 for frequency in the past month $(0=$ not at all or only one time, $1=$ once in a while, $2=$ one-half of the time, and $3=$ almost always). Item scores are summed to derive an overall severity score ranging from 0 to 51 . On the basis of prior administrations of this instrument, a score less than 10 indicates mild symptom severity, 11-20 indicates moderate, 21-35 signifies moderate to severe, and 36 and above indicates severe symptoms $[25,41,42]$. Severity scores for re-experiencing, avoidance, and arousal also were calculated by summing subscale items.

Risk for post-traumatic stress disorder: On the basis of prior studies of PTSS in childhood cancer survivors [28,43], DSM-IV diagnostic criteria for PTSD can be used for determining PTSS scores that may suggest risk of PTSD (yes/no). Risk of PTSD in this study was estimated by determining whether respondents met DSM-IV criteria A through F. All patients satisfied criterion A and E because their diagnosis and treatment constituted a 
traumatic event (criterion A), and the surveys for PTSS were conducted more than 1 month after initial cancer diagnosis (criterion $\mathrm{E}$, presence of symptoms for $>1$ month after the event). The PDS was used to assess PTSD criteria B, C, and D. Symptoms rated $\geq 1$ (once in a while) were counted as present for each PTSS symptom. To satisfy criterion $B$, the presence of at least one symptom related to re-experiencing was required. For criterion $\mathrm{C}$, the presence of at least three symptoms related to avoidance was required. Patients who endorsed presence of two symptoms related to arousal were considered to meet criterion D. Last, the Brief Symptoms Inventory-18 (BSI-18) [44] and the Medical Outcomes Study 36-Item Short Form Health Survey (SF-36) [45] were used to examine PTSD criterion F-the existence of significant distress and functional impairment, respectively. The BSI-18 consists of 18 items, yielding a global distress index (GSI) and three subscales of depression, anxiety, and somatization. Respondents indicated how much they had been bothered by each symptom over the previous 7 days, using a 5-point scale, ranging from 0 ('not at all') to 4 ('extremely'). Raw scores were converted to age-adjusted and gender-adjusted $T$-scores on the basis of adult nonpatient community norms. A GSI score of $\geq 63$ or a score of $\geq 63$ on any two of the three subscales signifies clinically significant distress (criterion F). BSI was administered at 6-month (Cronbach's $\alpha$ 0.90) and 12-month (Cronbach's $\alpha$ 0.93) follow-up. To assess impairment in functioning (PTSD criteria F), the SF-36 subscale assessing role limitations due to emotional health was used. A transformed $T$-score less than or equal to 40 indicates functional impairment. Respondents completed the SF-36 at baseline and at 12-month follow-up (Cronbach's $\alpha$ 0.91); therefore, statistical models examining a risk of PTSD using DSM-IV criteria were derived for 12-month follow-up only.

\section{Socio-demographic characteristics}

Self-reported socio-demographic variables were collected at baseline and included gender, race, employment/school status, and marital/relationship status. Age at diagnosis was categorized into three age groupings (14-19, 20-29, and 30-39 years) to estimate developmental life stages within this broad age range, using theoretical models of human development [46,47]. Patients reported their employment/school status at both baseline and prior to diagnosis, as well as at each subsequent time point of data collection. Respondents were categorized as 'employed/in school' when they were employed full-time/part time, fulltime homemaker, or full-time student. Those who were on temporary medical leave/disability, unemployed, or permanently unable to work were coded as 'unemployed/ not in school'. To evaluate the impact of employment/ school status on outcomes, we categorized patients into four groups: (i) employed/in school before diagnosis and at each subsequent time point; (ii) employed/in school before diagnosis but stopped at a subsequent time point without returning; (iii) unemployed/not in school before diagnosis and remained unemployed/not in school at each time point; and (iv) unemployed/not in school before diagnosis but initiated employment/school involvement at a subsequent time point.

\section{Clinical characteristics}

Through medical chart review, information about clinical characteristics obtained by the research staff included types of cancer, types of treatment, treatment status (on vs. off treatment), and types of surgery (e.g., treatmentrelated surgery, including bone marrow transplant, tumor resection, and central line placement; and non-treatment related such as staging procedures). Severity of disease was determined by using Surveillance Epidemiology and End Results codes to distinguish 'curable' forms of cancer from those with more uncertain prognosis [48]. Cluster 1 included diseases for which the 5-year survival rates are $90 \%$ or greater (Hodgkin's lymphoma, testicular tumors, and thyroid cancer) and account for $39 \%$ of all incident cases of invasive cancers in AYAs aged 15-39 years in the USA [49]. Cluster 2 was composed of all other invasive malignancies ( $61 \%$ of remaining incident cases in the USA) with expected 5-year survival rates of less than $90 \%$. Via self-report, a symptom checklist, derived by the investigators, elicited subject endorsement of 11 common treatment-related side effects (6-month follow-up only) (i.e. shortness of breath, memory or concentration problems, stomach or chest pain, ringing in ears, pain in joints, weight loss, frequent fevers, sleep difficulty, tiredness or fatigue, mouth sores, and frequent headaches), which were summed and considered in statistical analyses [48].

\section{Analytic strategies}

To identify the prevalence of PTSS over 1 year, we first examined descriptive statistics summarizing PTSS severity scores at 6-month and 12-month follow-up. We estimated the proportion of patients who were at risk of PTSD at 12-month follow-up. Changes in PTSS between 6-month and 12-month follow-up were tested by using a paired $T$-test and a Wilcoxon signed-rank test. A paired $T$-test was conducted to compare means of PTSS scores at 6 months with those at 12 months. A Wilcoxon signed-rank test was used to examine if respondents' categorical PTSS severity level had changed between 6-month and 12-month follow-up.

Next, we investigated predictors of changes in PTSS between 6-month and 12-month follow-up by using univariate and multivariate analyses. Repeated-measures ANOVAs were calculated for PTSS at 6-month and 12-month follow-up to examine whether change in respondents' PTSS scores were dependent upon sociodemographic and clinical characteristics. Last, multivariate linear regression was employed to investigate the predictive effects of socio-demographic and clinical characteristics on PTSS scores at 6-month and 12-month follow-up. On the basis of the results of repeated-measures ANOVAs, a $p$-value of $<0.20$ was used as a conservative criteria for selecting independent variables to include in multivariable models. Race and marital status were excluded in the regression model because $p$-values of both variables were greater than 0.20 in the univariate models. In the multivariate regression model, age at diagnosis, gender, employment/ school status, severity of disease, type of surgery, treatment status, and side effects were included as covariates. Type of treatment was excluded from analyses because virtually all respondents had received either chemotherapy or radiation. In regression analyses, 19 cases were dropped 
because of missing data in the measure of surgical types $(n=13)$, employment/school status $(n=6)$, and side effects $(n=2)$. A square-root transformation of PTSS scores was used in the analyses because the distribution for PTSS in this sample was highly negatively skewed. Summary scores of PTSS presented in tables were back transformed for interpretation. STATA v.11.2 (StataCorp. 2009. Stata Statistical Software: Release 11. College Station, TX: StataCorp LP) was used in analyzing the data.

\section{Results}

\section{Baseline characteristics}

Table 1 presents demographic and clinical characteristics of participants at baseline. Characteristics are provided for the overall group who participated at baseline $(n=215)$ and the subgroup who completed surveys at both 6-month and 12-month follow-up $(n=151)$. Among the 64 participants who did not complete follow-up surveys, 14 had died, 17 passively refused, and 33 responded to only one of the follow-up surveys. No significant differences in demographic and clinical characteristics were observed for these 64 patients lost to attrition and the 151 who completed data at all time points, except for age. Older patients were more likely to drop out than younger patients.

\section{The prevalence of PTSS and changes in PTSS over I year}

Mean and distribution of PTSS severity scores are summarized in Table 2. Mean PTSS scores are 11.09 at 6-month follow-up and 10.89 at 12-month follow-up $\left(t_{[150]}=0.90, p=0.36\right)$. Subscales of re-experiencing and avoidance show slight decreases between 6-month follow-up and 12-month follow-up $\left(t_{[145]}=1.78, p=0.08\right.$; $t_{[149]}=1.09, \quad p=0.28$, respectively), whereas arousal increases slightly $\left(t_{[145]}=-1.05, p=0.30\right)$. Median scores in PTSS were 9 for both 6-month and 12-month follow-up. About one-fourth of AYAs reported moderate levels of PTSS, $10 \%$ reported moderate to severe levels of PTSS, and 3\% reported severe levels of PTSS at 6-month follow-up. At 12-month follow-up, small but statistically non-significant increases in the proportion of moderate and moderate to severe levels of PTSS were observed $(p=0.15$ in signed-rank test). Twenty-nine percent of respondents' scores on the PDS, SF-36, and BSI-18 suggested risk of PTSD.

\section{The predictors of changes in PTSS over I year}

\section{Univariate analyses}

Table 3 presents results from repeated-measures ANOVAs. Only change in employment/school status after diagnosis appeared associated with changes in PTSS scores between 6-month and 12-month follow-up. Individuals who were unemployed/not in school before diagnosis reported increases in PTSS between 6-month and 12-month follow-up regardless of their current employment/school status. Those who were employed or in school before diagnosis but stopped their work or school reported almost no changes in PTSS scores. However, individuals who
Table I. Socio-demographic and clinical characteristics of participants

\begin{tabular}{|c|c|c|}
\hline & $\begin{array}{l}\text { Baseline } \\
(N=215)\end{array}$ & $\begin{array}{l}\text { Completed at } 6 \text { and } \\
12 \text { months }(N=151)\end{array}$ \\
\hline \multicolumn{3}{|l|}{ Socio-demographic characteristics } \\
\hline Age (baseline) & $23.7(8.8)$ & $22.9(9.0)$ \\
\hline \multicolumn{3}{|l|}{ Gender (baseline) } \\
\hline Female & $101(47.0 \%)$ & $68(45.0 \%)$ \\
\hline Male & II4 (53.0\%) & $83(55.0 \%)$ \\
\hline \multicolumn{3}{|l|}{ Race (baseline) } \\
\hline White/Caucasian & $95(44.6 \%)$ & $67(45.0 \%)$ \\
\hline Hispanic/Latino & $91(42.7 \%)$ & $68(45.6 \%)$ \\
\hline Others & $27(12.7 \%)$ & $14(9.4 \%)$ \\
\hline \multicolumn{3}{|l|}{ Education (baseline) } \\
\hline Less than high school & $102(47.9 \%)$ & $78(52.4 \%)$ \\
\hline High school graduate & $31(14.6 \%)$ & $18(12.1 \%)$ \\
\hline Some college & $45(21.1 \%)$ & $32(21.4 \%)$ \\
\hline College graduate and above & $35(16.4 \%)$ & $21(14.1 \%)$ \\
\hline \multicolumn{3}{|l|}{ Marital status (baseline) } \\
\hline Married/partnered & $81(39.4 \%)$ & $50(33.8 \%)$ \\
\hline Not married & $130(61.6 \%)$ & $98(66.2 \%)$ \\
\hline \multicolumn{3}{|c|}{ Employment/school status after diagnosis (12 months) } \\
\hline Remained employed/in school & $88(55.4 \%)$ & $81(55.5 \%)$ \\
\hline Stopped employment/school & $44(27.7 \%)$ & $40(27.4 \%)$ \\
\hline Remained unemployed/not in school & $12(7.6 \%)$ & $12(8.2 \%)$ \\
\hline Started employment/school & $15(9.4 \%)$ & $13(8.9 \%)$ \\
\hline \multicolumn{3}{|l|}{ Clinical characteristics } \\
\hline Age at diagnosis (baseline) & $23.6(8.9)$ & $22.7(9.0)$ \\
\hline \multicolumn{3}{|l|}{ Age group at diagnosis (baseline) } \\
\hline $14-19$ years & III (51.6\%) & $87(57.6 \%)$ \\
\hline 20-29 years & $39(18.2 \%)$ & $20(13.3 \%)$ \\
\hline 30-39 years & $65(30.2 \%)$ & $44(29.1 \%)$ \\
\hline \multicolumn{3}{|l|}{ Type of cancer (baseline) } \\
\hline Hodgkin's disease & $23(10.8 \%)$ & $16(10.6 \%)$ \\
\hline Non-Hodgkin's lymphoma & $17(7.9 \%)$ & $12(8.0 \%)$ \\
\hline Testicular cancer & $14(6.5 \%)$ & $7(4.6 \%)$ \\
\hline Female reproductive cancers & $8(3.7 \%)$ & $2(1.3 \%)$ \\
\hline Soft-tissue sarcoma & $21(9.8 \%)$ & $18(11.9 \%)$ \\
\hline Brain & $14(6.5 \%)$ & $12(8.0 \%)$ \\
\hline Leukemia & $50(23.4 \%)$ & $4 \mid(27.2 \%)$ \\
\hline Other carcinomas & $21(9.9 \%)$ & II (7.4\%) \\
\hline Breast & $19(8.9 \%)$ & $13(8.6 \%)$ \\
\hline Bone tumors & $26(12.2 \%)$ & $19(12.6 \%)$ \\
\hline \multicolumn{3}{|c|}{ Types of treatment (cumulative, 12 months) } \\
\hline Chemotherapy & 183 (93.9\%) & $139(92.1 \%)$ \\
\hline Radiation & $46(27.4 \%)$ & $33(27.9 \%)$ \\
\hline Surgery & $117(63.2 \%)$ & $84(55.6 \%)$ \\
\hline Bone marrow transplants & $15(7.0 \%)$ & $13(8.6 \%)$ \\
\hline \multicolumn{3}{|l|}{ Type of surgery (cumulative, 12 months) } \\
\hline $\begin{array}{l}\text { No surgery (chemotherapy or } \\
\text { radiation only) }\end{array}$ & 60 (35.3\%) & 60 (42.9\%) \\
\hline $\begin{array}{l}\text { Treatment-related surgery (including } \\
\text { bone marrow transplants, catheter } \\
\text { placement) }\end{array}$ & $92(54.1 \%)$ & $67(47.9 \%)$ \\
\hline Staging-related surgery & $18(10.6 \%)$ & $13(9.3 \%)$ \\
\hline \multicolumn{3}{|l|}{ Treatment status (at 12 months) } \\
\hline Stop treatment & $77(46.7 \%)$ & $67(44.4 \%)$ \\
\hline On active treatment & $88(53.3 \%)$ & $84(55.6 \%)$ \\
\hline $\begin{array}{l}\text { Number of side effects }(0 \sim 11) \\
\text { (at } 6 \text { months) }\end{array}$ & $3.53(2.47)$ & $3.50(2.49)$ \\
\hline
\end{tabular}

remain employed/in school after diagnosis reported slight decreases in PTSS scores. Finally, side effects measured at 6-month follow-up were significantly associated with levels of PTSS at 6-month follow-up (Pearson's correlation coefficient $0.43, p<0.001)$ and levels of PTSS at 12-month follow-up (Pearson's correlation coefficient $0.35, p<0.001)$. 
Table 2. Means and distributions of PTSS $(N=|5|)$

\begin{tabular}{|c|c|c|c|}
\hline & $N$ & $\begin{array}{l}\text { 6-month } \\
\text { follow-up }\end{array}$ & $\begin{array}{l}\text { I2-month } \\
\text { follow-up }\end{array}$ \\
\hline \multicolumn{4}{|l|}{ Severity scores } \\
\hline PTSS severity (0-5I) & $|5|$ & $11.09(9.87)^{\mathrm{a}}$ & $10.89(9.80)$ \\
\hline Re-experiencing $(0-15)$ & 146 & $3.16(3.45)$ & $2.93(3.40)$ \\
\hline Avoidance (0-2I) & 150 & $4.33(4.20)$ & $4.23(4.48)$ \\
\hline Arousal (0-15) & 146 & $3.70(3.67)$ & $3.77(3.36)$ \\
\hline \multicolumn{4}{|l|}{ Ordinal rating of severity } \\
\hline Mild $(0-10)$ & & $92(60.9 \%)^{b}$ & $84(55.6 \%)$ \\
\hline Moderate (I I-20) & & $38(25.2 \%)$ & $42(27.8 \%)$ \\
\hline Moderate to severe $(21-35)$ & & $16(10.6 \%)$ & $21(13.9 \%)$ \\
\hline Severe $(3-5 \mathrm{I})$ & & $5(3.3 \%)$ & $4(2.7 \%)$ \\
\hline \multicolumn{4}{|c|}{ Risk of PTSD based on the full DSM-IV criteria } \\
\hline At risk of PTSD & & - & $44(29.1 \%)$ \\
\hline Not at risk of PTSD & & - & $107(70.9 \%)$ \\
\hline
\end{tabular}

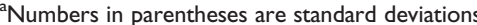

${ }^{b}$ Numbers presented first are frequencies, and numbers in the parentheses are percentages.

\section{Multivariate analyses}

Table 4 summarizes results from multivariate linear regression analyses inclusive of age at diagnosis, gender, employment/ school status, severity of cancer, type of surgery, and current treatment status as covariates. At 6-month follow-up, severity of PTSS was associated with side effects. Those who had greater number of side effects reported higher levels of PTSS. In addition, the association of cancer severity and PTSS was marginally significant: Patients with higher survival rate cancers reported higher levels of PTSS than did those with lower survival rate cancers. Socio-demographic factors and clinical characteristics explained $20.9 \%$ of variance in PTSS at 6-month follow-up.
At 12-month follow-up, 56.6\% of variance for PTSS was explained solely by PTSS at 6 months (Table 4). PTSS were significantly greater for patients with cancers of higher survival rates. Patients who received stagingrelated surgery reported significantly lower levels of PTSS than those who received treatment-related surgery. PTSS were significantly greater for patients who were on active treatment. Among socio-demographic variables, employment/ school status appeared as a significant predictor of PTSS at 12-month follow-up. Compared with AYAs who remained employed/in school throughout their diagnosis and treatment, patients who were not at all employed/in school reported significantly higher levels of PTSS. Disruption in employment/school work after diagnosis also was associated with higher levels of PTSS at 12-month follow-up, but at a marginally significant level.

\section{Discussion}

This study examined the prevalence and predictors of PTSS as well as changes in PTSS over the course of 1 year. The results showed that $44 \%$ of AYA patients reported at least moderate levels of PTSS approximately 1 year following their cancer diagnoses and 3\% experienced severe levels of PTSS. When the full criteria of DSM-IV were used to estimate the risk of PTSD at a 12-month follow-up, 29\% of AYAs reported risk of PTSD. The proportion of patients at risk of PTSD at 12 months post-diagnosis in this study is higher than that reported by Stuber et al. [28], who used the same method for estimated risk of PTSD as reported here. In their study of long-term survivors of childhood cancer, Stuber and

Table 3. Changes in transformed PTSS over time by subgroups

\begin{tabular}{|c|c|c|c|c|c|}
\hline & \multicolumn{2}{|c|}{ Mean (S.D.) ${ }^{\mathrm{a}}$} & \multirow{2}{*}{$\frac{\text { Group }}{p}$} & \multirow{2}{*}{$\frac{\text { Time }}{p}$} & \multirow{2}{*}{$\frac{\text { Group } \times \text { Time }^{b}}{p}$} \\
\hline & 6-month follow-up & I2-month follow-up & & & \\
\hline \multicolumn{6}{|l|}{ Age at diagnosis (categorical) $(n=|5|)$} \\
\hline $14-19$ years $(n=87)$ & $8.88(2.46)$ & $8.21(2.54)$ & 0.766 & 0.823 & $0.07 \mid$ \\
\hline $20-29$ years $(n=20)$ & $6.46(1.31)$ & $8.75(2.47)$ & & & \\
\hline $30-39$ years $(n=44)$ & $9.94(2.30)$ & $8.52(2.65)$ & & & \\
\hline \multicolumn{6}{|l|}{ Gender $(n=|5|)$} \\
\hline Male $(n=83)$ & $7.76(2.20)$ & $8.02(2.39)$ & & & \\
\hline \multicolumn{6}{|l|}{ Race $(n=149)$} \\
\hline White $(n=67)$ & $8.46(1.81)$ & $8.20(1.99)$ & 0.382 & 0.757 & 0.710 \\
\hline Non-White $(n=82)$ & $9.10(2.70)$ & $8.44(3.06)$ & & & \\
\hline \multicolumn{6}{|l|}{ Marital status (baseline) $(n=148)$} \\
\hline Not married/partnered $(n=98)$ & $8.63(2.34)$ & $8.12(2.51)$ & 0.707 & 0.366 & 0.985 \\
\hline Married/partnered $(n=50)$ & $9.19(2.28)$ & $8.69(2.70)$ & & & \\
\hline Remained employed/school $(n=8 \mathrm{I})$ & $8.79(2.03)$ & $7.11(2.61)$ & 0.560 & 0.175 & $<0.05$ \\
\hline Stopped employment/school $(n=40)$ & $10.20(2.79)$ & $10.27(2.54)$ & & & \\
\hline Remained unemployed/not in school $(n=12)$ & $6.42(3.00)$ & $10.18(2.80)$ & & & \\
\hline Started employment/school $(n=13)$ & $6.74(2.28)$ & $8.21(2.27)$ & & & \\
\hline \multicolumn{6}{|l|}{ Severity of disease $(n=|5|)$} \\
\hline Low survival rate $(n=126)$ & $8.74(2.41)$ & $7.93(2.47)$ & 0.381 & 0.687 & 0.110 \\
\hline High survival rate $(n=25)$ & $9.29(1.61)$ & $10.78(2.77)$ & & & \\
\hline \multicolumn{6}{|l|}{ Types of surgery (cumulative, 12-month) $(n=140$ ) } \\
\hline Treatment-related surgery $(n=67)$ & $9.10(2.88)$ & $8.45(2.59)$ & 0.793 & 0.160 & 0.329 \\
\hline Staging-related surgery $(n=13)$ & | $1.58(1.70)$ & $8.79(1.40)$ & & & \\
\hline No surgery (chemotherapy or radiation) $(n=60)$ & $8.18(1.83)$ & $8.43(2.80)$ & & & \\
\hline
\end{tabular}

${ }^{a}$ Mean and confidence intervals are back-transformed PTSS scores.

${ }^{\mathrm{b}}$ Repeated-measures ANOVAs were conducted on the basis of transformed scores for PTSS measures. 
Table 4. Linear regression analyses to predict PTSS at 6-month and I2-month follow-up $(n=132)$

\begin{tabular}{|c|c|c|c|c|}
\hline \multirow[b]{2}{*}{ Dependent variable } & \multicolumn{2}{|c|}{ PTSS at 6 months } & \multicolumn{2}{|c|}{ PTSS at I 2 months } \\
\hline & $\boldsymbol{\beta}$ & $p$ & $\boldsymbol{\beta}$ & $p$ \\
\hline PTSS at 6-month follow-up & & & 0.810 & $<0.001$ \\
\hline \multicolumn{5}{|l|}{ Age at diagnosis (categorical) } \\
\hline $14-19$ years & REF & & REF & \\
\hline 20-29 years & -0.382 & 0.322 & 0.314 & 0.272 \\
\hline 30-39 years & 0.112 & 0.687 & -0.053 & 0.795 \\
\hline \multicolumn{5}{|c|}{ Changes in employment/school status after diagnosis } \\
\hline Remained employed/school & REF & & REF & \\
\hline Stopped employment/school & 0.412 & 0.150 & 0.372 & 0.082 \\
\hline Remained unemployed/not in school & -0.572 & 0.139 & 0.710 & $<0.05$ \\
\hline Started employment/school & -0.631 & 0.353 & 0.414 & 0.212 \\
\hline Severity of disease ( $\mathrm{I}=$ high survival rate) & 0.764 & 0.067 & 1.07 & $<0.001$ \\
\hline \multicolumn{5}{|l|}{ Types of surgery (cumulative) } \\
\hline Staging-related surgery & -0.05 । & 0.916 & -0.940 & $<0.05$ \\
\hline No surgery (chemotherapy or radiation) & -0.147 & 0.573 & 0.052 & 0.776 \\
\hline Treatment status ( $\mathrm{I}$ = on treatment) & -0.085 & 0.762 & 0.499 & $<0.01$ \\
\hline Side effects at 6-month follow-up & 0.272 & $<0.00 \mid$ & -0.019 & 0.642 \\
\hline (Intercept) & 2.185 & $<0.001$ & -0.122 & 0.671 \\
\hline$R^{2}$ & 0.276 & & 0.665 & \\
\hline Adjusted $R^{2}$ & 0.209 & & 0.631 & \\
\hline F & 4.15 & $<0.001$ & 19.69 & $<0.001$ \\
\hline
\end{tabular}

colleagues estimated that $9 \%$ of childhood cancer survivors and $2 \%$ of siblings indicated risk of PTSD; however, the CCSS cohort is composed of survivors who were 15-34 years post-diagnosis. Other prior studies of long-term survivors of childhood cancer that used various methods to determine PTSD have reported rates of PTSD ranging from $3 \%$ to $21 \%[1,5,20-26]$. A study that examined PTSS in 30 children with cancer at 5 to 6 weeks after diagnosis found that $10 \%$ showed severe symptoms suggestive of PTSD [50]. Studies of older adult cancer patients in treatment found 2.4-22\% of patients indicating risk of PTSD by using a structured interview by clinicians or self-reported measures of PTSS [51,52]. In the study reported here, the mean PTSS scores for AYA patients at 6-month and 12-month follow-up were 11.09 and 10.89, respectively, which are again higher than the mean PTSS scores of young adult survivors reported in a previous study using the PDS $(M=6.6, S D=8.1, n=820)$ [53]. Differences in assessment methods, measures, sample sizes, years since diagnosis, treatment status (being on or off treatment), and sample characteristics across studies of young adult cancer survivors make it difficult to compare rates across studies and determine a definitive prevalence rate for PTSS in this population. Yet, the substantial portion of the sample in this study that reported moderate levels of PTSS as well as a potential risk of PTSD warrants further research and clinical attention in the AYA population.

The high levels of PTSS among AYA patients may be explained by a greater degree of vulnerability to traumatic stress during stages of adolescence and young adulthood $[2,33,47,54,55]$. When adolescents and young adult patients with cancer deal with issues related to normative developmental tasks and the need to cope with lifethreatening illnesses at the same time, these complex situations contribute to greater difficulties in reference to psychological adjustment [8-10,12-14]. Such difficulties may exacerbate their perceptions and experiences of their diagnoses of cancer and cancer-related treatments as traumatic events. However, this interpretation of the elevated PTSS in our data should be approached with caution. PTSS in this study was measured at 6 and 12 months after baseline surveys; more than half of the participants were still receiving some type of medical treatment at the 12-month assessment. Some of the PTSS symptoms may have been compounded by side effects, anxiety, or distress related to cancer treatment [4]. High rates of prevalence of PTSS may reflect normal reactions and responses to stressful situations, rather than indicating psychiatric conditions [7]. Furthermore, AYA patients undergoing cancer treatment in this study may still have been experiencing ongoing treatment as trauma at the time of assessment. The possibility of re-traumatization during treatment makes it unclear in terms of which event constitutes a trauma and which events patients perceive as traumatic $[7,56]$. Despite the lack of knowledge regarding specific traumatic events for patients in treatment, PTSS seems to appear among AYA patients during treatment and might be related to health behaviors and quality of life in the future. Future studies should be conducted to address these limitations by assessing traumatic aspects of cancer among those who had been recently diagnosed and in active treatment, the impact of traumatic aspects of cancer and PTSS that deal with developmental tasks for AYA patients with cancer, and longitudinal changes in their traumatic responses.

Findings also indicated that PTSS manifested as early as 6 months post-diagnosis and did not increase or decrease significantly thereafter. This finding is consistent with work carried out by Phipps and colleagues [35] in which similar levels of PTSS were observed in childhood cancer survivors 6 to 18 months post-diagnosis. However, these findings contrast with published longitudinal research on older adult cancer patients and families of cancer patients, in which PTSS decreased within a relatively short period [51,57-60]. 
Findings revealed that those who maintained employment/ school attendance after diagnosis experienced significantly lower levels of PTSS at 12-month follow-up compared with those who were not employed or in school before and after diagnosis. This result suggests that being unemployed/not in school before diagnosis may be a marker of other stressors, such as financial strain, which is associated with increases in PTSS. This finding is also consistent with previous studies indicating the correlations between unemployment and distress $[1,27,29,31,36]$. In addition, those who stopped work or school after diagnosis reported marginally significantly higher levels of PTSS at 12-month follow-up than those who were employed/in school after diagnosis, suggesting the importance of maintaining AYA's involvement in work or school to the extent possible. Remaining occupied in school or work may provide AYAs with a social support system and sense of control over their lives that serve to buffer the traumatic aspects of cancer and its treatment. Alternatively, maintaining involvement in school or work may simply function as a behavioral indicator of people who are not substantially affected by cancer-induced PTSS and/or side effects in the first place.

In terms of clinical characteristics and their relationship to PTSS, the finding that reporting side effects was associated with PTSS is consistent with the previous studies of long-term survivors of childhood cancer [29], which found that survivors who had worse health conditions and lower levels of physical health-related quality of life showed more PTSS than those who did not. As pain and worsening health conditions resulting from cancer treatment are often regarded by patients as the most frightening aspects in the cancer process [61], it is possible that poor physical symptoms and health conditions are experienced as reoccurring traumas that elevate the risk of developing PTSS. In addition, we note the counter-intuitive finding that AYAs with high survival rate cancers indicated higher levels of PTSS than did those with low survival rate cancers. This finding conflicts with childhood cancer survivor studies in which severity of illness was not observed to be related to PTSS [62]. This finding may imply that severity of disease is relevant only to the extent to which AYA patients perceive cancer as traumatic. It may be that AYAs with a high potential for survival still struggle in reconciling fears about their future with oncologists assurances that they will be 'cured' or that the type of cancer they have is 'a good kind of cancer' relative to those with a worse prognosis. It also is possible that patients with high survival rate cancers have less contact with medical staff than other cancer patients because of their shorter periods of treatment and follow-up, and thus experience less support and encouragement from their oncology care providers. This lack of perceived support during the course of illness may lead to an estimation of their perceiving cancer and its treatment as more traumatic when compared with AYAs with low survival-rate cancers. Future research is needed to examine how changes in the perceptions of a diagnosis of cancer and its treatment over time differ depending on the severity of the cancer. Types of surgery that patients received were significantly related to PTSS at 12-month follow-up. Patients who received surgical treatment reported greater PTSS than those who received staging-related surgery. This finding suggests that surgical treatment for cancer is more physically invasive compared with staging-related surgeries and therefore can be perceived as more traumatic for patients. Last, treatment status appeared as a significant predictor for PTSS at 12-month follow-up. Treatment may not only serve as a traumatic event for cancer patients but also as a constant reminder of traumatic events related to cancer experienced in the past. Thus, patients who were on active treatment report greater PTSS.

The findings of this study illustrate the fact that a relatively high rate of AYA patients with cancer experience clinically significant levels of PTSS in the first year of diagnosis and that the levels of PTSS stabilize from 6 to 12 months after diagnosis. These findings emphasize the importance of early screening and intervention for PTSS among AYA patients with cancer as early as the first 6 months following diagnosis. As existing interventions targeting PTSS among AYAs with cancer focus on longterm survivors of childhood cancer or families of children recently diagnosed with cancer [63-65], data from this study suggest that preventive interventions to alleviate PTSS should be developed for recently diagnosed AYAs. The risk factors found in this study, such as employment/ school status before and after diagnosis, severity of disease, type of surgery, current treatment status, and side effects, can be used to identify and screen people who practice high-risk activities among recently diagnosed AYA patients and monitor over time.

This study's strengths include its moderately sized and ethnically diverse multi-institutional sample of AYAs recruited for this prospective, longitudinal study relatively close to the time of their initial cancer diagnoses, along with a high participation rate $(75 \%)$ and equal gender distribution. A well-validated measure of PTSS was utilized at two key time points across the survivorship continuum. The study is somewhat limited by the study design, which does not include a control group. The inclusion of a control group would be helpful in terms of estimating in a more accurate and rigorous way to what extent PTSS is elevated by cancer or cancer-related factors. In addition, the measure for risk of PTSD was limited because we did not assess participants' reactions to traumatic events (i.e., reactions involving intense fear, helplessness, or horror), which refers to the PTSD criteria A2 in DSM-IV. This study assumed that all participants would satisfy this criterion, but this may not have been true for some participants. Finally, the study did not include data on the subjective appraisal of traumatic events and trauma history before the diagnosis of cancer. As previous research identified subjective appraisals and prior histories of traumatic events as the most influential predictive factors for PTSS and PTSD [33,51,66,67], future studies should include investigations of lifetime trauma history and the subjective appraisal of traumatic events such as being diagnosed with cancer and receiving medical treatment.

This study provides evidence of PTSS in AYA cancer patients. It furthers our understanding of factors contributing to increased risks of distress in the AYA population. Future research should further develop clinical guidelines that can help health-care professionals in pediatric or adult settings to sensitively respond to the traumatic stress experienced by AYA patients. In doing so, health care 
professionals may be able to assess distress and needs related to traumatic stress in the early stages of cancer treatment, and provide appropriate support to AYA patients at risk of PTSD. Trauma-focused cognitive and behavioral therapies are demonstrated as effective in reducing distress [68,69]. Integrating them into peer support models that are developmentally appropriate for AYAs may prove fruitful in terms of reducing distress and promoting quality of life. Future research that further investigates the nature of PTSS and develops effective care for ongoing PTSS among AYA patients would improve the psychosocial adjustment of AYA patients with cancer.

\section{Acknowledgement}

Research support was provided by HopeLab Foundation, Redwood City, California.

\section{References}

1. Stuber ML, Kazak, AE, Meeske $\mathrm{K}$ et al. Predictors of posttraumatic stress symptoms in childhood cancer survivors. Pediatrics 1997;100:958-964.

2. Akechi T, Okuyama T, Sugawara Y, Nakano T, Shima Y, Uchitomi Y. Major depression, adjustment disorders, and post-traumatic stress disorder in terminally ill cancer patients: Associated and predictive factors. J Clin Oncol 2004;22:1957-1965.

3. Boyer BA, Bubel D, Jacobs SR et al. Posttraumatic stress in women with breast cancer and their daughters. Am J Fam Ther 2002;30:323-338.

4. Kangas M, Henry JL, Bryant RA. Posttraumatic stress disorder following cancer: a conceptual and empirical review. Clin Psychol Rev 2002;22:499-524.

5. Bruce M. A systematic and conceptual review of posttraumatic stress in childhood cancer survivors and their parents. Clin Psychol Rev 2006;26:233-256.

6. American Psychiatric Association. Diagnostic and statistical manual of mental disorders: DSM-IV. American Psychiatric Association: Washington, DC, 1994.

7. Kazak AE, Boeving CA, Alderfer MA, Hwang WT, Reilly A. Posttraumatic stress symptoms during treatment in parents of children with cancer. $J$ Clin Oncol 2005;23:7405-7410.

8. Evan E, Zeltzer LK. Psychosocial dimensions of cancer in adolescents and young adults. Cancer 2006;107:1663-1671.

9. Roberts C, Turney M, Knowles A. Psychosocial issues of adolescents with cancer. Soc Work Health Care 1998;27:3-18.

10. Zebrack BJ, Chesler ML, Penn A. Psychosocial support. In Cancer in Adolescents and Young Adults, Bleyer WA, Barr RD (eds). Springer: Berlin Heidelberg, 2007; 375-385.

11. Albritton K, Bleyer W. The management of cancer in the older adolescent. Eur J Cancer 2003;39:2584-2599.

12. Zeltzer L. Cancer in adolescents and young adults psychosocial aspects: long-term survivors. Cancer 1993;71:3463-3468.

13. Zebrack BJ. Psychological, social, and behavioral issues for young adults with cancer. Cancer 2011;117:2289-2294.

14. D'Agostino NM, Penney A, Zebrack BJ. Providing developmentally appropriate psychosocial care to adolescent and young adult cancer survivors. Cancer 2011;117(10 Suppl):23292334.

15. Stava CJ, Lopez A. Health profiles of younger and older breast cancer survivors. Cancer 2006;107:1752-1759.

16. Ganz PA, Schag CC, Heinrich RL. The psychosocial impact of cancer on the elderly: a comparison with younger patients. $J$ Am Geriatr Soc 1985;33:429-435.

17. Vinokur AD, Threatt BA, Vinokur-Kaplan D, Satariano WA. The process of recovery from breast cancer for younger and older patients: changes during the first year. Cancer 1990; 65:1242-1254.

18. Mor V, Allen S, Malin M. The psychosocial impact of cancer on older versus younger patients and their families. Cancer 1994;74:2118-2127.

19. Davis LL. Posttraumatic stress disorder in children and adolescents: a review and analysis. Clin Child Fam Psychol Rev 2000;3:135-154.

20. Taïeb O, Moro MR, Baubet T, Revah-Lévy A, Flament MF. Posttraumatic stress symptoms after childhood cancer. Eur Child Adolesc Psychiatry 2003;12:255-264.

21. Ozono S, Saeki T, Mantani T, Ogata A, Okamura H, Yamawaki S. Factors related to posttraumatic stress in adolescent survivors of childhood cancer and their parents. Support Care Cancer 2007;15:309-317.

22. Butler RW, Rizzi LP, Handwerger BA. Brief report: the assessment of posttraumatic stress disorder in pediatric cancer patients and survivors. J Pediatr Psychol 1996;21:499-504.

23. Erickson SJ, Steiner H. Trauma and personality correlates in long term pediatric cancer survivors. Child Psychiatry Hum Dev 2001;31:195-213.

24. Kazak AE, Barakat LP, Alderfer M et al. Posttraumatic stress in survivors of childhood cancer and mothers: development and validation of the Impact of Traumatic Stressors Interview Schedule (ITSIS). J Clin Psychol Med Settings 2001;8:307-323.

25. DeKeyser Ganz F, Raz H, Gothelf D, Yaniv I, Buchval I. Post-traumatic stress disorder in Israeli survivors of childhood cancer. Oncol Nurs Forum 2010;37:160-167.

26. Kazak AE, Alderfer M, Rourke MT, Simms S, Streisand R, Grossman JR. Posttraumatic stress disorder (PTSD) and posttraumatic stress symptoms (PTSS) in families of adolescent childhood cancer survivors. J Pediatr Psychol 2004;29:211-219.

27. Rourke MT, Hobbie WL, Schwartz L, Kazak AE. Posttraumatic stress disorder (PTSD) in young adult survivors of childhood cancer. Pediatr Blood Cancer 2007;49:177-182.

28. Stuber ML, Meeske KA, Krull KR et al. Prevalence and predictors of posttraumatic stress disorder in adult survivors of childhood cancer. Pediatrics 2010;125:E1124-E1134.

29. Langeveld NE, Grootenhuis MA, Voûte PA, de Haan RJ. Posttraumatic stress symptoms in adult survivors of childhood cancer. Pediatr Blood Cancer 2004;42:604-610.

30. Schwartz L, Drotar D. Posttraumatic stress and related impairment in survivors of childhood cancer in early adulthood compared to healthy peers. J Pediatr Psychol 2005;31:356-366.

31. Lee YL, Santacroce SJ. Posttraumatic stress in long-term young adult survivors of childhood cancer: a questionnaire survey. Int J Nurs Stud 2007;44:1406-1417.

32. Taylor N, Absolon K, Snowden J, Eiser C. Need for psychological follow-up among young adult survivors of childhood cancer. Eur J Cancer Care 2011;21:52-58.

33. Hobbie WL, Stuber M, Meeske $\mathrm{K}$ et al. Symptoms of posttraumatic stress in young adult survivors of childhood cancer. $J$ Clin Oncol 2000;18:4060-4066.

34. Pelcovitz D, Libov BG, Mandel F, Kaplan S, Weinblatt M, Septimus A. Posttraumatic stress disorder and family functioning in adolescent cancer. J Trauma Stress 1998;11:205-221.

35. Phipps S, Long A, Hudson M, Rai SN. Symptoms of post-traumatic stress in children with cancer and their parents: effects of informant and time from diagnosis. Pediatr Blood Cancer 2005;45:952-959.

36. Schrag NM, McKeown RE, Jackson KL, Cuffe SP, Neuberg RW. Stress-related mental disorders in childhood cancer survivors. Pediatr Blood Cancer 2008;50:98-103.

37. Wu E, Robinson LL, Jenney MEM et al. Assessment of health-related quality of life of adolescent cancer patients using the Minneapolis-Manchester Quality of Life Adolescent Questionnaire. Pediatr Blood Cancer 2007;48:678-686.

38. Hedström M, Ljungman G, von Essen L. Perceptions of distress among adolescents recently diagnosed with cancer. $J$ Pediatr Hematol Oncol 2005;27:15-22.

39. Enskär K, von Essen L. Prevalence of aspects of distress, coping, support and care among adolescents and young adults undergoing and being off cancer treatment. Eur J Oncol Nurs 2007;11:400-408.

40. von Essen L, Enskär K, Kreuger A, Larsson B, Sjödén PO. Self-esteem, depression and anxiety among Swedish children and adolescents on and off cancer treatment. Acta Paediatr 2000;89:229-236.

41. Foa EB, Cashman L, Jaycox L, Perry K. The validation of a self-report measure of posttraumatic stress disorder: the Posttraumatic Diagnostic Scale. Psychol Assess 1997;9:445-451.

42. Marx BP, Sloan DM. Peritraumatic dissociation and experiential avoidance as predictors of posttraumatic stress symptomatology. Behav Res Ther 2005;43:569-583.

43. Stuber ML, Meeske KA, Leisenring W et al. Defining medical post-traumatic stress among young adult survivors in the childhood cancer survivor study. Gen Hosp Psychiatry 2011;33 (4):347-353.

44. Derogatis LR. Brief Symptom Inventory: BSI; Administration, Scoring, and Procedures 
Manual. National Computer Systems: Minneapolis, MN, 1993.

45. Ware JE, Snow KK, Kosinski M, Gandek B. SF-36 Health Survey: Manual and Interpretation Guide. The Health Institute, New England Medical Center: Boston, 1993.

46. Oktay JS, Walter CA. Breast Cancer in the Life Course: Women's Experiences. Springer: New York, 1991.

47. Arnett JJ. Emerging adulthood: a theory of development from the late teens through the twenties. Am Psychol 2000;55:469-480.

48. Zebrack BJ, Block R, Hayes-Lattin B et al. Psychosocial service use and unmet need among recently diagnosed adolescent and young adult cancer patients. Cancer 2012, DOI: 10.1002/cncr.27713

49. Howlader N, Noone AM, Krapcho M, et al. SEER Cancer Statistics Review, 1975-2008, November 2010.

50. Landolt MA, Vollrath M, Ribi K, Gnehm HE Sennhauser FH. Incidence and associations of parental and child posttraumatic stress symptoms in pediatric patients. J Child Psychol Psychiatry 2003:44:1199-1207.

51. Mehnert A, Koch U. Prevalence of acute and post-traumatic stress disorder and comorbid mental disorders in breast cancer patients during primary cancer care: a prospective study. Psycho-Oncology 2007;16:181-188.

52. Kangas M, Henry JL, Bryant RA. The relationship between acute stress disorder and posttraumatic stress disorder following cancer. J Consult Clin Psychol 2005;73:360-364.

53. Seitz DCM, Besier T, Debatin K et al. Posttraumatic stress, depression and anxiety among adult long-term survivors of cancer in adolescence. Eur J Cancer 2010;46:1596-1606.
54. Maercker A, Michael T, Fehm L, Becker ES, Margraf J. Age of traumatisation as a predictor of post-traumatic stress disorder or major depression in young women. $\mathrm{Br} J$ Psychiatry 2004;184:482-487.

55. Flanagan C, Reuman D, Buchanan CM et al. Development during adolescence: the impact of stage-environment fit on young adolescents' experiences in schools and in families. Am Psychol 1993;48:90-101.

56. Kazak AE, Derosa BW, Schwartz LA et al. Psychological outcomes and health beliefs in adolescent and young adult survivors of childhood cancer and controls. J Clin Oncol 2010;28:2002-2007.

57. Pöder U, Ljungman G, von Essen, L. Posttraumatic stress disorder among parents of children on cancer treatment: a longitudinal study. Psycho-Oncology 2008;17:430-437.

58. Dolgin MJ, Phipps S, Fairclough DL et al. Trajectories of adjustment in mothers of children with newly diagnosed cancer: a natural history investigation. $J$ Pediatr Psychol 2007;32:771-782

59. Cernvall M, Alaine I, von Essen L. The factor structure of traumatic stress in parents of children with cancer: a longitudinal analysis. J Pediatr Psychol 2011;1-10.

60. Koopman C, Butler LD, Classen C et al. Traumatic stress symptoms among women with recently diagnosed primary breast cancer. $J$ Trauma Stress 2002;15:277-287.

61. Kazak AE, Prusak A, McSherry M et al. The Psychosocial Assessment Tool (PAT): pilot data on a brief screening instrument for identifying high risk families in pediatric oncology. Fam Syst Health 2001;19:303-317.
62. Brown RT, Madan-Swain A, Lambert R Posttraumatic stress symptoms in adolescen survivors of childhood cancer and their mothers. J Trauma Stress 2003;16:309-318.

63. Kazak AE. Evidence-based interventions for survivors of childhood cancer and their families. $J$ Pediatr Psychol 2005;30:29-39.

64. Kazak AE, Alderfer MA, Streisand R et al Treatment of posttraumatic stress symptoms in adolescent survivors of childhood cancer and their families: a randomized clinical trial. J Fam Psychol 2004;18:493-504.

65. Kazak AE, Simms S, Alderfer MA et al. Feasibility and preliminary outcomes from a pilot study of a brief psychological intervention for families of children newly diagnosed with cancer. $J$ Pediatr Psychol 2005;30:644-655

66. Stuber ML, Meeske K, Gonzalez S, Houskamp BM, Pynoos R. Post-traumatic stress after childhood cancer I: the role of appraisal. Psycho-Oncology 1994;3:305-312.

67. Widows MR, Jacobsen PB, Fields KK Relation of psychological vulnerability factors to posttraumatic stress disorder symptomatology in bone marrow transplant recipients. Psychosom Med 2000;62:873-882.

68. Cohen JA, Mannarino AP, Berliner L, Deblinger E. Trauma-focused cognitive behavioral therapy for children and adolescents: an empirical update. $J$ Interpers Violence 2000;15:1202-1223.

69. Bradley R, Greene J, Russ E, Dutra L, Westen D. A multidimensional meta-analysis of psychotherapy for PTSD. Am $J$ Psychiatry 2005;162:214-227. 